\title{
Understanding Gender Dynamics in Fruit Cultivation under Indian Condition
}

\author{
Sahu Ankita ${ }^{1 *}$, Kishore Kundan ${ }^{2}$, J.C. Jeeva ${ }^{1}$, S.K. Srivastava ${ }^{1}$ and \\ Mohapatra Lalatendu ${ }^{3}$ \\ ${ }^{1}$ (Fruit Science), Senior Scientist (Agricultural Extension) and Director ICAR-Central \\ Institute for Women in Agriculture, Bhubaneswar 751003, India \\ ${ }^{2}$ (Fruit Science) ICAR-IIHR Central Horticultural Experiment Station, \\ Bhubaneswar 751019, India \\ ${ }^{5}$ Department of Agriculture and Farmer's Empowerment Govt. of Odisha, India
}

*Corresponding author

\section{A B S T R A C T}

\section{Keywords \\ Gender, Farm women, Fruit, Cultivation, Gender gap \\ Article Info \\ Accepted: \\ 15 September 2018 Available Online: 10 October 2018}

The study aims at identifying the potential role of Indian farmwomen in fruit based production system along with realising their constraints which limits their considerable contribution in terms of technology application, use of agri-inputs and commercial marketing. The magnitude of their participation is hardly visualized, which creates perceptible gender gap. The emerging issues of farmer suicide, rural-urban migration of male members and disenchantment of rural youth in agriculture, overburden the Indian farmwomen with farming activities. Gender analysis will help in formulating suitable strategies for providing better platform to women farmers to realize their untapped potential in bring sustainability to the production system.

\section{Introduction}

Indian women farmers are vital entities for guaranteeing food security not only for their families but also for the community (Oluwatayo, 2012). Their roles vary from region to region and are transforming rapidly in many parts of India, where economic and social forces are revolutionizing the agricultural sector (Sekhar et al., 2013). The roles and status of women in agriculture and rural areas vary widely with region, age, literacy rates, ethnicity and social class
(Fartyal and Rathore, 2013). They play a substantial role in production system, encompassing cultivation, crop management, harvesting, maintenance, sorting, grading, packaging and selling.

They form a magnitude of work force in agriculture encompassing 79 per cent as against only 63 per cent of men (NSSO, 2010). In rural areas the percentage of women work force is even higher, 84 per cent (Lal and Khurana, 2011). Majority of farm women are overburdened, face enormous drudgery and occupational health hazards in their workplace 
and are economically, nutritionally under secured. They have poor exposure to advanced agricultural technologies, Govt. schemes and policies and even lack the essential prerequisites to avail the subsidy facilities in agriculture due to lack of ownership of land.

They often face harassment in terms of lower wage rate, poor working facilities and are often ignored and overlooked due to the nature of work they perform which is often regarded as non-skilful, monotonous and time consuming. In order to sustain these vulnerable communities, there is a need to quantify and showcase their potential and irreplaceable role. As well as identify the constraints, which the society has created for them, so that suitable strategies can be thought of in order to bring them to a platform where they have independency to execute and realize their untapped potential in bringing sustainability to the production system. Fruit cultivation is highly remunerative and can ensure stabilization of women's empowerment by providing employment opportunities through commercial cultivation, post-harvest management \& processing, nursery production, agri-horti tourism etc. Therefore, an attempt has been made in this study to assess women's latent role, gender gaps and constraints in fruit based production system.

\section{Materials and Methods}

The investigation was carried out during 2016 in Kuchinda block of Sambalpur district of Odisha, India, by interviewing 138 farmwomen selected by stratified random sampling. Nine villages having potential fruit growing areas were selected for the purpose of study. The responses of 138 farmwomen on various aspects of fruit cultivation were collected through semi-structured interview schedule. The various activities associated with fruit cultivation is listed in Table 2.

The farm women engaged in fruit cultivation were asked to assess their participation in each of these activities and score was allotted accordingly based on following parameters.

Crop wise frequency of respondents in 1-4 categories in each activity (1-32) was worked out and percentage of participation was calculated. Objective quantification of participation of woman in each parameter was done by defining clearly the full, moderate, low and nominal participation allotting weighed scores and calculating the participation quotient. Participation quotient was calculated by using a 0-3 scale of weighted score. Category of nominal participation was allotted 0 , low participation 1, moderate participation 2 and full participation 3 weighted score. The participation quotient was calculated by using following formula.

Mean score of participation Participation quotient = ---------------- X 100 Maximum obtainable score of participation

The participation quotients were further used for working out mean, standard deviation, variance, co-efficient of variation and standard error mean. Based on participation quotient, the extent of participation was worked out activity-wise and crop-wise into four categories based on following scale.

\section{Results and Discussion}

The farm women's participation in various activities of fruit crops was surveyed on the basis of comparative participation scale (1-4) consisting of four categories as nil, low, moderate and full. Their category wise percentage participation in different crops and activities has been presented in Table 1 . Activity wise over all participation quotients, Crop wise participation quotients and Crop wise over all participation quotients of farm women in various activities of fruit cultivation 
have been presented in Table 2, 3 and 4 respectively. Based on the findings of the survey (as per Table 1, 2, 3 and 4), women's role/participation in various aspects of fruit cultivation has been discussed under following heads:

\section{Field preparation and use of various farm machineries}

Farm women had moderate participation in land preparation and layout of fruit crops like mango (54.60 c), banana (62.96 c), papaya $(55.56 \mathrm{c})$ and litchi $(35.56 \mathrm{c})$. The participation in use of machineries for spraying operation was nominal (29.00 a), while in case of other farm machineries and tools it was low (36.93 b). Mango, banana and litchi were commercial fruit crops of the region. Due to abundance in availability and lower wage rate, the magnitude of women labourers was more in the orchard. As a result, such drudgery prone activities in the orchards were equally shared by both men and women. Use of power operated machineries were solely conducted by male counterparts as only men received training and thus acquired the skill in operation of power operated machineries such as $\mathrm{s}$ tractor, tree pruner, power tiller and power sprayer. Such activities were exclusively done by men due to two main reason firstly the stereotype nature of the society that considered such activities to be male centric, including the manufacturers and distributors, who designed and promoted the models which were not gender neutral. Even the spraying operations were mostly carried out by men as most of the women labourers accompanied their infants and children in farm activities and were apprehensive about the occupational health hazards encountered during spraying of agro-chemicals. As a point of intervention, it is suggested that gender neutral machineries should be designed and promoted in a country like India where the abundance of female workers is quite acute.
Empowerment of women farmers in use of machineries that are low cost, mostly solar operated and easy to maintain can bring revolution in Indian agriculture. Moreover the subsidy pattern of Govt. in availing these machineries makes it unfeasible for women farmers in accessing such machineries. However with suitable policy interventions such inputs can be extended to women groups. Suitable arrangements of shelter places for the infants and provision of drinking water, food will encourage and motivate the women farmers in their work place.

\section{Production aspects}

The farm women had full participation in post planting care (63.78 d), mulching (60.39 d) and harvesting $(77.07 \mathrm{~d})$. While, the participation was moderate in planting of fruit crops $(56.38 \mathrm{c})$, application of organic manures $(49.67 \mathrm{c})$, water management (44.06 c) and weed management $(47.66 \mathrm{c})$. There was full participation in planting of mango (65.52 d) and banana $(81.48 \mathrm{~d})$ due to increase in cropping area of these crops under different Govt. schemes. It was observed that both men and women had equal role to play in production aspects of fruit crops. However, the nature of the society, orthodox mindset of people and gender blindness compel us to overlook the unique role of women farmers. Farm women are often regarded as labourers and not as a growers.

\section{Knowledge and participation on improved technologies of fruit crops}

Farm women had nominal to low knowledge regarding improved technologies of fruit crops. In activities like high density planting (28.25 a), training, pruning (29.71 a) and modern ripening techniques (23.53 a), they had nominal knowledge. While, in activities like nursery raising techniques (33.61 b), drip irrigation $(33.21 \mathrm{~b})$, fertilizer application, 
nutrient scheduling (39.53 b), knowledge of major pest, diseases $(35.11 \mathrm{~b})$ and integrated pest and disease management (40.08 b), they had low level of knowledge and participation. They possessed moderate knowledge regarding critical stages of irrigation (51.28 c) and full knowledge on fruit maturity $(61.01 \mathrm{~d})$. The farm women had full participation in planting material production of mango (60.92 d), moderate participation in high density planting (HDP) in mango (52.87 c) and banana $(74.07 \mathrm{c})$. It was observed that women of the region had poor technical competency. However with government interventions in the form of schemes and policies, women farmers had certain level of knowledge in improved production technologies of commercially viable fruit crops like mango and banana. Limited knowledge and participation of farm women in improved technologies of fruit crops can be attributed to the fact that there existed huge gender gap due to differential level of exposure of men and women to various training, skill up gradation and capacity building programmes. Similar findings were also obtained by Sethi and Sharma 2011, as they reported that the involvement of women in production-oriented trainings and other extension programmes should be not only as beneficiaries but also as change agents for higher adoption and diffusion of latest farm technologies.

\section{Post-production aspects}

Farm women had moderate participation in sorting \& grading of fruits $(51.22 \mathrm{c})$, low participation in value addition, preservation and processing of fruits $(37.00 \mathrm{~b})$ and nominal participation in marketing of processed products $(25.43$ a). The participation of women in marketing of fresh fruits was better in papaya $(86.12 \mathrm{~d})$, guava $(83.34 \mathrm{~d})$, lemon $(83.34 \mathrm{~d})$ and minor fruits $(72.73 \mathrm{~d})$ as these fruits were mostly sold in local markets. However, in commercially important fruit crops like mango (55.17 c), banana (61.12 b) and litchi $(33.34 \mathrm{c})$, the male members dominated over marketing of fruits to distant markets, as these fruits fetched better prices and were harvested in bulk. Due to lack of proper post-harvest infrastructure facilities, the processing of fruit crops and the marketing of processed products was low and nominal respectively.

\section{Level of participation}

\begin{tabular}{|c|l|l|}
\hline Sl. no. & Level of participation & Parameter \\
\hline $\mathbf{1}$ & Full participation & Women participation is more than men \\
\hline $\mathbf{2}$ & Moderate participation & Equal participation by both men and women \\
\hline $\mathbf{3}$ & Low participation & Involvement of women is less \\
\hline $\mathbf{4}$ & No participation & Poor participation by women \\
\hline
\end{tabular}

The extent of participation was worked out activity-wise and crop-wise into four categories

\begin{tabular}{|c|l|c|l|}
\hline SI. No. & \multicolumn{1}{|c|}{ Category } & Symbol & \multicolumn{1}{c|}{ Calculated values } \\
\hline $\mathbf{1}$ & No participation & (a) & $<($ General Mean-1SD) \\
\hline $\mathbf{2}$ & Low participation & (b) & $<$ General Mean and $>($ General Mean-1SD) \\
\hline $\mathbf{3}$ & Moderate participation & (c) & $<($ General Mean+1SD) and $>$ General Mean \\
\hline $\mathbf{4}$ & Full participation & (d) & $\begin{array}{l}<(\text { General Mean+2SD) and }>(\text { General } \\
\text { Mean+1SD })\end{array}$ \\
\hline
\end{tabular}




\section{Int.J.Curr.Microbiol.App.Sci (2018) 7(10): 1923-1933}

Table.1 Crop wise per cent participation of farm women in different activities of fruit cultivation

\begin{tabular}{|c|c|c|c|c|c|c|c|c|c|c|c|c|c|c|c|c|c|c|}
\hline \multirow[t]{2}{*}{ Crop } & \multirow[b]{2}{*}{ 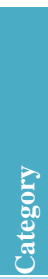 } & \multirow{2}{*}{ 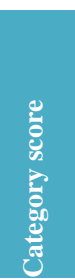 } & \multicolumn{16}{|c|}{ Crop wise per cent participation of farm women in different activities of fruit cultivation (Activity 1-16) } \\
\hline & & & 豙 & 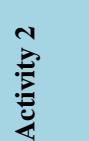 & $\sum_{\frac{2}{2}}^{m}$ & 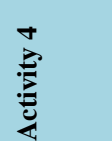 & 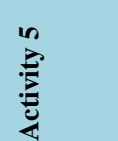 & 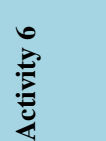 & $\sum_{\substack{\frac{2}{2} \\
\frac{2}{2}}}^{n}$ & $\sum_{\substack{i\\
}}^{\infty}$ & $\sum_{\vec{Z}}^{a}$ & 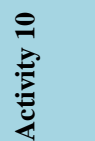 & 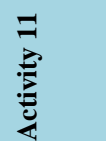 & $\frac{2}{2}$ & $\frac{m}{2}$ & 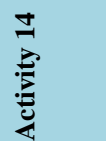 & $\frac{n}{3}$ & $\frac{0}{3}$ \\
\hline \multirow[t]{4}{*}{ Mango } & 1 & 0 & 5.17 & 6.89 & 22.41 & 12.07 & 3.44 & 6.89 & 3.44 & 5.17 & 8.62 & 20.68 & 12.06 & 25.86 & 3.44 & 13.79 & 3.44 & 12.06 \\
\hline & 2 & 1 & 17.24 & 32.75 & 29.31 & 24.14 & 37.93 & 36.20 & 31.03 & 15.51 & 46.55 & 36.20 & 44.82 & 41.37 & 46.55 & 36.20 & 44.82 & 41.38 \\
\hline & 3 & 2 & 55.17 & 20.68 & 31.03 & 32.76 & 50.00 & 51.72 & 31.03 & 55.17 & 22.41 & 17.24 & 17.24 & 32.76 & 41.38 & 24.14 & 43.10 & 22.41 \\
\hline & 4 & 3 & 22.41 & 39.65 & 17.24 & 31.03 & 8.62 & 5.17 & 34.48 & 24.13 & 22.41 & 25.86 & 25.86 & 0 & 8.62 & 25.86 & 8.62 & 24.14 \\
\hline \multirow[t]{4}{*}{$\overline{\text { Banana }}$} & 1 & 0 & 11.12 & 0 & 16.67 & 16.67 & 0 & 0 & 5.56 & 0 & 5.56 & 16.67 & 16.67 & 33.34 & 0 & 22.23 & 0 & 11.12 \\
\hline & 2 & 1 & 5.56 & 22.23 & 11.12 & 5.56 & 16.67 & 22.23 & 5.56 & 22.23 & 27.78 & 11.12 & 11.12 & 5.56 & 22.23 & 0 & 22.23 & 11.12 \\
\hline & 3 & 2 & 72.23 & 11.12 & 66.67 & 11.12 & 77.78 & 72.23 & 27.78 & 61.12 & 5.56 & 11.12 & 11.12 & 61.12 & 72.23 & 16.67 & 72.23 & 16.67 \\
\hline & 4 & 3 & 11.12 & 66.67 & 5.56 & 66.67 & 5.56 & 5.56 & 61.12 & 16.67 & 61.12 & 61.12 & 61.12 & 0 & 5.56 & 61.12 & 5.56 & 61.12 \\
\hline \multirow[t]{4}{*}{ Litchi } & 1 & 0 & 20.00 & 26.67 & 46.67 & 53.34 & 13.34 & 13.34 & 6.67 & 13.34 & 33.34 & 20.00 & 53.34 & 40.00 & 13.34 & 26.67 & 13.34 & 0 \\
\hline & 2 & 1 & 53.34 & 46.67 & 40.00 & 20.00 & 66.67 & 73.34 & 73.34 & 20.00 & 66.67 & 60.00 & 13.34 & 40.00 & 40.00 & 33.34 & 46.67 & 53.34 \\
\hline & 3 & 2 & 26.67 & 26.67 & 13.34 & 26.67 & 20.00 & 13.34 & 20.00 & 60.00 & 0 & 20.00 & 33.34 & 13.34 & 33.34 & 40.00 & 33.34 & 40.00 \\
\hline & 4 & 3 & 0 & 0 & 0 & 0 & 0 & 0 & 0 & 6.67 & 0 & 0 & 0 & 6.67 & 13.34 & 0 & 6.67 & 6.67 \\
\hline \multirow[t]{4}{*}{ Papaya } & 1 & 0 & 0 & 16.67 & 0 & 100.00 & 0 & 8.34 & 0 & 0 & 50.00 & 50.00 & 0 & 33.34 & 0 & 0 & 0 & $\overline{0}$ \\
\hline & 2 & 1 & 33.34 & 50.00 & 50.00 & 0 & 41.67 & 41.67 & 25.00 & 0 & 50.00 & 50.00 & 66.67 & 66.67 & 41.67 & 75.00 & 16.67 & 41.67 \\
\hline & 3 & 2 & 33.34 & 25.00 & 50.00 & 0 & 50.00 & 33.34 & 58.34 & 33.34 & 0 & 0 & 33.34 & 0 & 41.67 & 25.00 & 83.34 & 50.00 \\
\hline & 4 & 3 & 33.34 & 8.34 & 0 & 0 & 8.34 & 16.67 & 16.67 & 66.67 & 0 & 0 & 0 & 0 & 16.67 & 0 & 0 & 8.34 \\
\hline \multirow[t]{4}{*}{ Guava } & 1 & 0 & 0 & 30.00 & 30.00 & 0 & 0 & 0 & 0 & 0 & 40.00 & 30.00 & 20.00 & 10.00 & 20.00 & 20.00 & 40.00 & $\overline{0}$ \\
\hline & 2 & 1 & 40.00 & 40.00 & 60.00 & 30.00 & 80.00 & 40.00 & 60.00 & 10.00 & 60.00 & 60.00 & 50.00 & 50.00 & 20.00 & 60.00 & 60.00 & 40.00 \\
\hline & 3 & 2 & 20.00 & 30.00 & 10.00 & 70.00 & 20.00 & 60.00 & 40.00 & 60.00 & 0 & 10.00 & 30.00 & 40.00 & 60.00 & 20.00 & 0 & 60.00 \\
\hline & 4 & 3 & 40.00 & 0 & 0 & 0 & 0 & 0 & 0 & 30.00 & 0 & 0 & 0 & 0 & 0 & 0 & 0 & 0 \\
\hline \multirow[t]{4}{*}{ Lemon } & 1 & 0 & 21.43 & 14.29 & 28.57 & 57.14 & 21.43 & 42.86 & 7.14 & 21.43 & 64.29 & 14.29 & 50.00 & 21.43 & 0 & 28.57 & 7.14 & 0 \\
\hline & 2 & 1 & 28.57 & 64.29 & 71.43 & 42.86 & 71.43 & 50.00 & 78.57 & 57.14 & 35.71 & 64.29 & 42.86 & 78.57 & 57.14 & 57.14 & 50.00 & 71.43 \\
\hline & 3 & 2 & 42.86 & 21.43 & 0 & 0 & 7.14 & 7.14 & 14.29 & 21.43 & 0 & 21.43 & 7.14 & 0 & 42.86 & 14.29 & 42.86 & 28.57 \\
\hline & 4 & 3 & 7.14 & 0 & 0 & 0 & 0 & 0 & 0 & 0 & 0 & 0 & 0 & 0 & 0 & 0 & 0 & 0 \\
\hline \multirow{4}{*}{$\begin{array}{l}\text { Minor } \\
\text { fruits }\end{array}$} & 1 & 0 & 18.18 & 63.64 & 54.55 & 90.91 & 90.91 & 27.27 & 0 & 0 & 100.00 & 63.64 & 54.55 & 72.73 & 0 & 72.73 & 27.27 & 18.18 \\
\hline & 2 & 1 & 45.45 & 36.36 & 45.45 & 9.09 & 9.09 & 72.73 & 9.09 & 0 & 0 & 36.36 & 45.45 & 27.27 & 100.00 & 27.27 & $\mathbf{7 2 . 7 3}$ & 81.82 \\
\hline & 3 & 2 & 27.27 & 0 & 0 & 0 & 0 & 0 & 90.91 & 100.00 & 0 & 0 & 0 & 0 & 0 & 0 & 0 & 0 \\
\hline & 4 & 3 & 9.09 & 0 & 0 & 0 & 0 & 0 & 0 & 0 & 0 & 0 & 0 & 0 & 0 & 0 & 0 & 0 \\
\hline
\end{tabular}


Crop wise per cent participation of farm women in different activities of fruit cultivation (Activity 17-32) cont...

\begin{tabular}{|c|c|c|c|c|c|c|c|c|c|c|c|c|c|c|c|c|c|c|}
\hline Crop & نั & 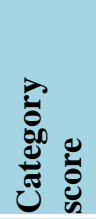 & ב & 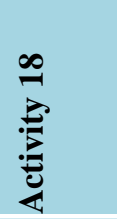 & $\frac{2}{2}$ & 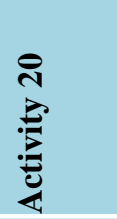 & 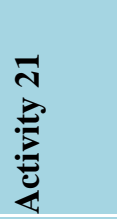 & 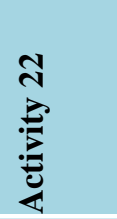 & 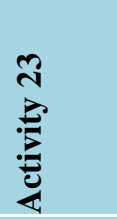 & 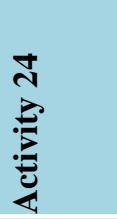 & 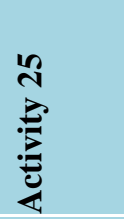 & 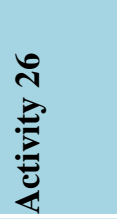 & 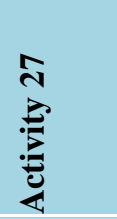 & 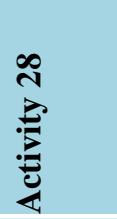 & 芳 & 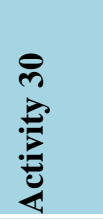 & 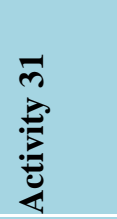 & $\frac{N}{3}$ \\
\hline \multirow[t]{4}{*}{ Mango } & 1 & 0 & 32.75 & 24.14 & 13.79 & 13.79 & 41.38 & 34.48 & 31.03 & 0 & 15.51 & 29.31 & 6.89 & 12.07 & 12.07 & 17.24 & 22.41 & 31.03 \\
\hline & 2 & 1 & 29.31 & 34.48 & 48.28 & 27.59 & 15.51 & 31.03 & 27.59 & 37.93 & 34.48 & 25.86 & 43.10 & 25.86 & 39.66 & 29.31 & 32.76 & 24.14 \\
\hline & 3 & 2 & 13.79 & 17.24 & 12.06 & 31.03 & 17.24 & 24.14 & 34.48 & 31.03 & 43.10 & 17.24 & 44.82 & 46.55 & 20.69 & 27.59 & 18.97 & 17.24 \\
\hline & 4 & 3 & 24.14 & 24.14 & 25.86 & 27.59 & 25.86 & 10.34 & 6.90 & 31.03 & 6.89 & 27.58 & 5.17 & 15.52 & 27.59 & 25.86 & 25.86 & 27.59 \\
\hline \multirow[t]{4}{*}{ Banana } & 1 & 0 & 22.23 & 22.23 & 22.23 & 16.67 & 22.23 & 11.12 & 16.67 & 0 & 11.12 & 22.23 & 5.56 & 11.12 & 11.12 & 100 & 100 & 22.23 \\
\hline & 2 & 1 & 5.56 & 5.56 & 5.56 & 11.12 & 27.78 & 16.67 & 16.67 & 16.67 & 16.67 & 11.12 & 27.78 & 5.56 & 11.12 & 0 & 0 & 5.56 \\
\hline & 3 & 2 & 5.56 & 5.56 & 16.67 & 11.12 & 27.78 & 61.12 & 66.67 & 16.67 & 72.23 & 27.78 & 61.12 & 72.23 & 16.67 & 0 & 0 & 5.56 \\
\hline & 4 & 3 & 66.67 & 66.67 & 55.56 & 61.12 & 22.23 & 11.12 & 0 & 66.67 & 0 & 38.89 & 5.56 & 11.12 & 61.12 & 0 & 0 & 66.67 \\
\hline \multirow{4}{*}{ Litchi } & 1 & 0 & 73.34 & 0 & 26.67 & 33.34 & 46.67 & 40.00 & 66.67 & 6.67 & 0 & 60.00 & 13.34 & 46.67 & 46.67 & 33.34 & 100.00 & 6.67 \\
\hline & 2 & 1 & 13.34 & 33.34 & 60.00 & 33.34 & 33.34 & 60.00 & 13.34 & 33.34 & 66.67 & 40.00 & 40.00 & 6.67 & 20.00 & 66.67 & 0 & 66.67 \\
\hline & 3 & 2 & 13.34 & 60.00 & 6.67 & 26.67 & 20.00 & 0 & 20.00 & 53.34 & 33.34 & 0 & 46.67 & 46.67 & 33.34 & 0 & 0 & 20.00 \\
\hline & 4 & 3 & 0 & 6.67 & 6.67 & 6.67 & 0 & 0 & 0 & 6.67 & 0 & 0 & 0 & 0 & 0 & 0 & 0 & 6.67 \\
\hline \multirow[t]{4}{*}{ Papaya } & 1 & 0 & 0 & 0 & 16.67 & 25.00 & 33.34 & 16.67 & 0 & 0 & 0 & 50.00 & 0 & 0 & 0 & 0 & 0 & 8.34 \\
\hline & 2 & 1 & 66.67 & 16.67 & 58.34 & 50.00 & 58.34 & 41.67 & 0 & 0 & 0 & 50.00 & 25.00 & 0 & 58.34 & 41.67 & 25.00 & 50.00 \\
\hline & 3 & 2 & 33.34 & 58.34 & 25.00 & 25.00 & 8.34 & 41.67 & 66.67 & 16.67 & 41.67 & 0 & 50.00 & 41.67 & 33.34 & 58.34 & 58.34 & 41.67 \\
\hline & 4 & 3 & 0 & 25.00 & 0 & 0 & 0 & 0 & 33.34 & 83.34 & 58.34 & 0 & 25.00 & 58.34 & 8.34 & 0 & 16.67 & 0 \\
\hline \multirow[t]{4}{*}{ Guava } & 1 & 0 & 10.00 & 0 & 50.00 & 30.00 & 30.00 & 10.00 & 0 & 0 & 0 & 80.00 & 0 & 0 & 0 & 20.00 & 30.00 & 40.00 \\
\hline & 2 & 1 & 70.00 & 50.00 & 40.00 & 50.00 & 70.00 & 70.00 & 40.00 & 0 & 0 & 20.00 & 20.00 & 0 & 80.00 & 50.00 & 70.00 & 50.00 \\
\hline & 3 & 2 & 20.00 & 50.00 & 10.00 & 20.00 & 0 & 20.00 & 60.00 & 20.00 & 70.00 & 0 & 80.00 & 50.00 & 20.00 & 30.00 & 0 & 10.00 \\
\hline & 4 & 3 & 0 & 0 & 0 & 0 & 0 & 0 & 0 & 80.00 & 30.00 & 0 & 0 & 50.00 & 0 & 0 & 0 & 0 \\
\hline \multirow[t]{4}{*}{ Lemon } & 1 & 0 & 50.00 & 0 & 21.43 & 0 & 28.57 & 14.29 & 14.29 & 0 & 0 & 78.57 & 0 & 0 & 0 & 0 & 21.43 & 35.71 \\
\hline & 2 & 1 & 50.00 & 35.71 & 64.29 & 71.43 & 57.14 & 64.29 & 57.14 & 0 & 42.86 & 21.43 & 28.57 & 7.14 & 28.57 & 21.43 & 78.57 & 57.14 \\
\hline & 3 & 2 & 0 & 50.00 & 14.29 & 28.57 & 14.29 & 21.43 & 28.57 & 57.14 & 57.14 & 0 & 50.00 & 35.71 & 71.43 & 50.00 & 0 & 7.14 \\
\hline & 4 & 3 & 0 & 14.29 & 0 & 0 & 0 & 0 & 0 & 42.86 & 0 & 0 & 21.43 & 57.14 & 0 & 28.57 & 0 & 0 \\
\hline \multirow[t]{4}{*}{ Minor fruits } & 1 & 0 & 81.82 & 0 & 72.73 & 72.73 & 72.73 & 9.09 & 0 & 0 & 0 & 63.64 & 45.45 & 0 & 9.09 & 63.64 & 63.64 & 45.45 \\
\hline & 2 & 1 & 18.18 & 0 & 27.27 & 27.27 & 27.27 & 90.91 & 27.27 & 27.27 & 0 & 36.36 & 54.55 & 9.09 & $\mathbf{5 4 . 5 5}$ & 18.18 & 27.27 & 54.55 \\
\hline & 3 & 2 & 0 & 100.00 & 0 & 0 & 0 & 0 & 72.73 & 36.36 & 100.00 & 0 & 0 & 63.64 & 36.36 & 0 & 9.09 & 0 \\
\hline & 4 & 3 & 0 & & 0 & 0 & 0 & 0 & 0 & 36.36 & 0 & 0 & 0 & 27.27 & 0 & 18.18 & 0 & 0 \\
\hline
\end{tabular}


Table.2 Activity wise over all participation quotients of farm women in fruit cultivation

\begin{tabular}{|c|c|c|}
\hline Code & Aspect & Mean Participation quotient \\
\hline Activity-1 & Decision in selecting the type of crops to be grown & $54.65 \mathrm{c}$ \\
\hline Activity-2 & Involvement in selection of fruit crops and their varieties & $43.14 \mathrm{~b}$ \\
\hline Activity-3 & Access to quality planting material & $34.18 \mathrm{~b}$ \\
\hline Activity-4 & Involvement in nursery raising & $33.61 \mathrm{~b}$ \\
\hline Activity-5 & Involvement in land preparation and layout & $40.04 \mathrm{~b}$ \\
\hline Activity-6 & Involvement in pit digging and filling & $42.56 \mathrm{~b}$ \\
\hline Activity-7 & Involvement in planting & $56.38 \mathrm{c}$ \\
\hline Activity-8 & Involvement in post planting care & $63.78 \mathrm{~d}$ \\
\hline Activity-9 & Knowledge and skill on High Density Planting & $28.25 \mathrm{a}$ \\
\hline Activity-10 & Selection of intercrops & $35.16 \mathrm{~b}$ \\
\hline Activity-11 & Involvement in purchase of agricultural inputs (planting materials, fertilizers, insecticides and pesticides etc.) & $38.07 \mathrm{~b}$ \\
\hline Activity-12 & Knowledge and participation in canopy management of fruit crops & $29.71 \mathrm{a}$ \\
\hline Activity-13 & Knowledge and participation in application of organic manure & $49.67 \mathrm{c}$ \\
\hline Activity-14 & Knowledge and participation in application of inorganic fertilizers & $39.53 \mathrm{~b}$ \\
\hline Activity-15 & Knowledge and participation in water management & $44.06 \mathrm{c}$ \\
\hline Activity-16 & Knowledge on critical stages of irrigation & $51.28 \mathrm{c}$ \\
\hline Activity-17 & Knowledge on drip irrigation & $33.21 \mathrm{~b}$ \\
\hline Activity-18 & Mulching & $60.39 \mathrm{~d}$ \\
\hline Activity-19 & Knowledge of important pest and diseases & $35.11 \mathrm{~b}$ \\
\hline Activity-20 & Knowledge and practice of integrated pest and disease management & $40.08 \mathrm{~b}$ \\
\hline Activity-21 & Use of machineries for spraying operation & $29.00 \mathrm{a}$ \\
\hline Activity-22 & Use of other farm machineries and tools & $36.93 \mathrm{~b}$ \\
\hline Activity-23 & Weed Management & $47.66 \mathrm{c}$ \\
\hline Activity-24 & Involvement in harvesting of matured fruits & $77.07 \mathrm{~d}$ \\
\hline Activity-25 & Knowledge on fruit maturity & $61.01 \mathrm{~d}$ \\
\hline Activity-26 & Knowledge and skills in modern ripening technique & $23.53 \mathrm{a}$ \\
\hline Activity-27 & Sorting and grading of fruits & $51.22 \mathrm{c}$ \\
\hline Activity-28 & Involvement in marketing & $67.87 \mathrm{~d}$ \\
\hline Activity-29 & Decision in case of surplus yield and glut in market & $49.85 \mathrm{c}$ \\
\hline Activity-30 & Value addition, preservation and processing of fresh fruits & $37.00 \mathrm{~b}$ \\
\hline Activity-31 & Marketing of processed products & $25.43 \mathrm{a}$ \\
\hline \multirow[t]{6}{*}{ Activity-32 } & Access to training, skill up gradation and capacity building programs & $38.76 \mathrm{~b}$ \\
\hline & Mean & 43.69 \\
\hline & SD & 12.74 \\
\hline & Variance & 162.31 \\
\hline & $\mathbf{C V}$ & 0.29 \\
\hline & SEm & 1.08 \\
\hline
\end{tabular}


Table.3 Crop wise participation quotients of farm women in various activities of fruit cultivation

\begin{tabular}{|c|c|c|c|c|c|c|c|c|c|}
\hline & \multicolumn{9}{|c|}{ Fruit crop wise participation quotient in each activity* } \\
\hline & Mango & Banana & Litchi & Papaya & Guava & Lemon & Minor fruits & Mean & SD \\
\hline Activity 1 & $64.95 \mathrm{~d}$ & $61.11 \mathrm{~b}$ & $35.56 \mathrm{c}$ & $66.67 \mathrm{c}$ & $66.67 \mathrm{~d}$ & $45.24 \mathrm{c}$ & $42.42 \mathrm{c}$ & 54.65 & 12.18 \\
\hline Activity 2 & $64.37 \mathrm{~d}$ & $81.48 \mathrm{~d}$ & $33.34 \mathrm{c}$ & $41.67 \mathrm{~b}$ & $33.34 \mathrm{~b}$ & $35.71 \mathrm{~b}$ & $12.12 \mathrm{~b}$ & 43.14 & 21.15 \\
\hline Activity 3 & $47.70 \mathrm{~b}$ & $53.70 \mathrm{~b}$ & $22.23 \mathrm{~b}$ & $50.00 \mathrm{~b}$ & $26.67 \mathrm{~b}$ & $23.81 \mathrm{~b}$ & $15.15 b$ & 34.18 & 14.56 \\
\hline Activity 4 & $60.92 \mathrm{~d}$ & $75.93 \mathrm{c}$ & $24.45 \mathrm{~b}$ & $0 \mathrm{a}$ & $56.67 \mathrm{c}$ & $14.29 \mathrm{a}$ & $3.03 \mathrm{a}$ & 33.61 & 28.26 \\
\hline Activity 5 & $54.60 \mathrm{c}$ & $62.96 \mathrm{c}$ & $35.56 \mathrm{c}$ & $55.56 \mathrm{c}$ & $40.00 \mathrm{~b}$ & $28.57 \mathrm{~b}$ & $3.03 \mathrm{a}$ & 40.04 & 18.90 \\
\hline Activity 6 & $51.72 \mathrm{~b}$ & $61.11 \mathrm{~b}$ & $33.34 \mathrm{c}$ & $52.78 \mathrm{c}$ & $53.34 \mathrm{c}$ & $21.43 b$ & $24.24 b$ & 42.56 & 14.72 \\
\hline Activity 7 & $65.52 \mathrm{~d}$ & $81.48 \mathrm{~d}$ & $37.78 \mathrm{c}$ & $63.89 \mathrm{c}$ & $46.67 \mathrm{c}$ & $35.71 \mathrm{~b}$ & $63.64 \mathrm{~d}$ & 56.38 & 15.54 \\
\hline Activity 8 & $66.09 \mathrm{~d}$ & $64.81 \mathrm{c}$ & $53.34 \mathrm{~d}$ & $88.89 \mathrm{~d}$ & $73.34 \mathrm{~d}$ & $33.34 \mathrm{~b}$ & $66.67 \mathrm{~d}$ & 63.78 & 15.91 \\
\hline Activity 9 & $52.87 \mathrm{c}$ & $74.07 \mathrm{c}$ & $22.23 \mathrm{~b}$ & $16.67 \mathrm{a}$ & $20.00 \mathrm{a}$ & $11.91 \mathrm{a}$ & $0 \mathrm{a}$ & 28.25 & 23.93 \\
\hline Activity 10 & $49.43 \mathrm{~b}$ & $72.23 \mathrm{c}$ & $33.34 \mathrm{c}$ & $16.67 \mathrm{a}$ & $26.67 \mathrm{~b}$ & $35.71 \mathrm{~b}$ & $12.12 \mathrm{~b}$ & 35.16 & 19.00 \\
\hline Activity 11 & $52.30 \mathrm{c}$ & $72.23 \mathrm{c}$ & $26.67 \mathrm{~b}$ & $44.45 \mathrm{~b}$ & $36.67 \mathrm{~b}$ & $19.05 \mathrm{a}$ & $15.15 \mathrm{~b}$ & 38.07 & 18.63 \\
\hline Activity 12 & $35.63 \mathrm{a}$ & $42.59 \mathrm{a}$ & $28.89 \mathrm{~b}$ & $22.23 \mathrm{a}$ & $43.34 \mathrm{c}$ & $26.19 \mathrm{~b}$ & $9.09 \mathrm{~b}$ & 29.71 & 11.22 \\
\hline Activity 13 & $51.72 \mathrm{~b}$ & $61.12 \mathrm{~b}$ & $48.89 \mathrm{~d}$ & $58.34 \mathrm{c}$ & $46.67 \mathrm{c}$ & $47.62 \mathrm{c}$ & $33.34 \mathrm{c}$ & 49.67 & 8.37 \\
\hline Activity 14 & $54.02 \mathrm{c}$ & $72.23 \mathrm{c}$ & $37.78 \mathrm{c}$ & $41.67 \mathrm{~b}$ & $33.34 \mathrm{~b}$ & $28.57 \mathrm{~b}$ & $9.09 \mathrm{~b}$ & 39.53 & 18.40 \\
\hline Activity 15 & $52.30 \mathrm{c}$ & $61.12 \mathrm{~b}$ & $44.45 \mathrm{c}$ & $61.12 \mathrm{c}$ & $20.00 \mathrm{a}$ & $45.24 \mathrm{c}$ & $24.24 \mathrm{~b}$ & 44.06 & 15.22 \\
\hline Activity 16 & $52.87 \mathrm{c}$ & $75.93 \mathrm{c}$ & $51.12 \mathrm{~d}$ & $55.56 \mathrm{c}$ & $53.34 \mathrm{c}$ & $42.86 \mathrm{c}$ & $27.27 \mathrm{~b}$ & 51.28 & 13.52 \\
\hline Activity 17 & $43.10 \mathrm{a}$ & $72.23 \mathrm{c}$ & $13.34 \mathrm{a}$ & $44.45 \mathrm{~b}$ & $36.67 \mathrm{~b}$ & $16.67 \mathrm{a}$ & $6.06 \mathrm{~b}$ & 33.21 & 21.26 \\
\hline Activity 18 & $47.13 b$ & $72.23 \mathrm{c}$ & $57.78 \mathrm{~d}$ & $69.45 \mathrm{c}$ & $50.00 \mathrm{c}$ & $59.53 \mathrm{~d}$ & $66.67 \mathrm{~d}$ & 60.39 & 8.89 \\
\hline Activity 19 & $50.00 \mathrm{~b}$ & $68.52 \mathrm{c}$ & $31.12 \mathrm{~b}$ & $36.12 \mathrm{~b}$ & $20.00 \mathrm{a}$ & $30.95 \mathrm{~b}$ & $9.09 \mathrm{~b}$ & 35.11 & 18.04 \\
\hline Activity 20 & $57.47 \mathrm{c}$ & $72.23 \mathrm{c}$ & $35.56 \mathrm{c}$ & $33.34 \mathrm{~b}$ & $30.00 \mathrm{~b}$ & $42.86 \mathrm{c}$ & $9.09 \mathrm{~b}$ & 40.08 & 18.79 \\
\hline Activity 21 & $42.53 \mathrm{a}$ & $50.00 \mathrm{~b}$ & $24.45 \mathrm{~b}$ & $25.00 \mathrm{a}$ & $23.34 \mathrm{~b}$ & $28.57 \mathrm{~b}$ & $9.09 \mathrm{~b}$ & 29.00 & 12.48 \\
\hline Activity 22 & $36.78 \mathrm{a}$ & $57.40 \mathrm{~b}$ & $20.00 \mathrm{~b}$ & $41.67 \mathrm{~b}$ & $36.67 \mathrm{~b}$ & $35.71 \mathrm{~b}$ & $30.30 \mathrm{c}$ & 36.93 & 10.51 \\
\hline Activity 23 & $39.08 \mathrm{a}$ & $50.00 \mathrm{~b}$ & $17.78 \mathrm{a}$ & $77.78 \mathrm{~d}$ & $53.34 \mathrm{c}$ & $38.10 \mathrm{c}$ & $57.58 \mathrm{~d}$ & 47.66 & 17.33 \\
\hline Activity 24 & $64.37 \mathrm{~d}$ & $83.34 \mathrm{~d}$ & $53.34 \mathrm{~d}$ & $94.45 \mathrm{~d}$ & $93.34 \mathrm{~d}$ & $80.95 \mathrm{~d}$ & $69.70 \mathrm{~d}$ & 77.07 & 14.14 \\
\hline Activity 25 & $47.13 b$ & $53.70 \mathrm{~b}$ & $44.45 \mathrm{c}$ & $86.12 \mathrm{~d}$ & $76.67 \mathrm{~d}$ & $52.38 \mathrm{c}$ & $66.67 \mathrm{~d}$ & 61.01 & 14.65 \\
\hline Activity 26 & $47.70 \mathrm{~b}$ & $61.12 \mathrm{~b}$ & $13.34 \mathrm{a}$ & $16.67 \mathrm{a}$ & $6.67 \mathrm{a}$ & $7.14 \mathrm{a}$ & $12.12 \mathrm{~b}$ & 23.53 & 20.11 \\
\hline Activity 27 & $49.43 \mathrm{~b}$ & $55.56 \mathrm{~b}$ & $44.45 \mathrm{c}$ & $66.67 \mathrm{c}$ & $60.00 \mathrm{c}$ & $64.28 \mathrm{~d}$ & $18.18 \mathrm{~b}$ & 51.22 & 15.33 \\
\hline Activity 28 & $55.17 \mathrm{c}$ & $61.12 b$ & $33.34 \mathrm{c}$ & $86.12 \mathrm{~d}$ & $83.34 \mathrm{~d}$ & $83.34 \mathrm{~d}$ & $72.73 \mathrm{~d}$ & 67.87 & 17.87 \\
\hline Activity 29 & $54.60 \mathrm{c}$ & $75.93 \mathrm{c}$ & $28.89 \mathrm{~b}$ & $50.00 \mathrm{~b}$ & $40.00 \mathrm{~b}$ & $57.14 \mathrm{~d}$ & $42.42 \mathrm{c}$ & 49.85 & 13.87 \\
\hline Activity 30 & $54.02 \mathrm{c}$ & $0 \mathrm{a}$ & $22.23 \mathrm{~b}$ & $52.78 \mathrm{c}$ & $36.67 \mathrm{~b}$ & $69.05 \mathrm{~d}$ & $24.24 \mathrm{~b}$ & 37.00 & 21.77 \\
\hline Activity 31 & $49.43 \mathrm{~b}$ & $0 \mathrm{a}$ & $0 \mathrm{a}$ & $63.89 \mathrm{c}$ & $23.34 \mathrm{~b}$ & $26.19 \mathrm{~b}$ & $15.15 \mathrm{~b}$ & 25.43 & 22.23 \\
\hline Activity 32 & $47.13 b$ & $72.23 \mathrm{c}$ & $42.23 \mathrm{c}$ & $44.45 \mathrm{~b}$ & $23.34 \mathrm{~b}$ & $23.81 \mathrm{~b}$ & $18.18 \mathrm{~b}$ & 38.76 & 17.40 \\
\hline Mean & 51.94 & 61.86 & 32.85 & 50.78 & 42.81 & 37.87 & 27.75 & 43.69 & \\
\hline SD & 7.83 & 18.78 & 12.99 & 22.67 & 19.98 & 18.68 & 22.78 & & \\
\hline
\end{tabular}

*a-nominal participation, b-low participation, c-moderate participation, d-full participation 
Int.J.Curr.Microbiol.App.Sci (2018) 7(10): 1923-1933

Table.4 Crop wise over all participation quotients of farm women in fruit cultivation

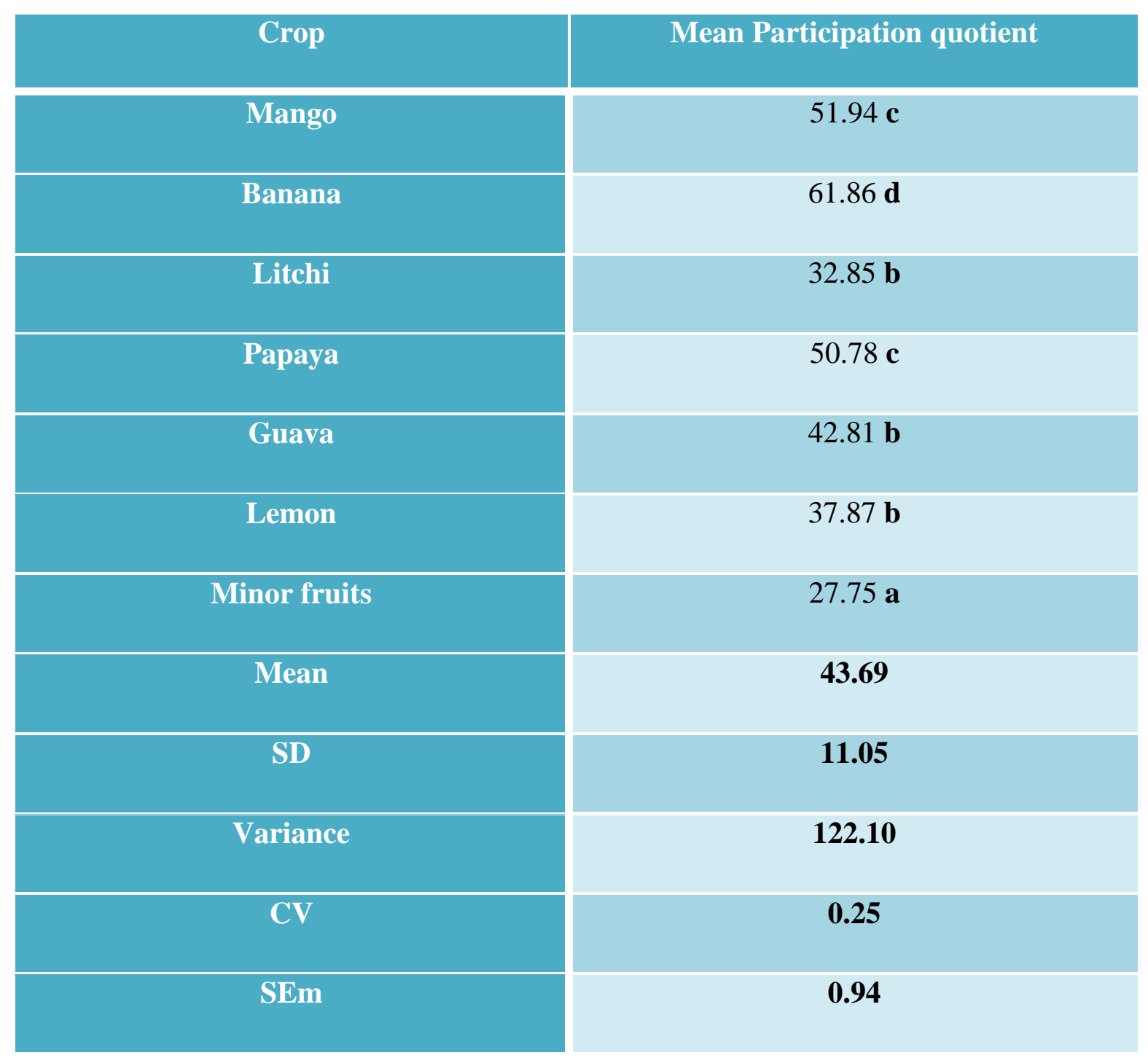

*a-nominal participation, b-low participation, c-moderate participation, d-full participation 
The involvement of farm women in post-harvest management of fruit crops was poor on account of small and marginal farm holdings, lack of training on post-harvest handling, inconsistent post-harvest management practices and poor infrastructure, including cold storage facilities.

\section{Access to resources}

The access of farm women to several resources refer to the ease in obtaining various inputs required for cultivation of fruit crops viz., land and other agro inputs such as planting materials, farm tools and implements, organic manures, inorganic fertilizers, pesticides and growth regulators etc. The various training and capacity building programmes held at village/block/district level was also considered as a source of technical resource. The availability of quality planting material was low in case of all fruit crops. However, the participation of farm women in purchase of agricultural inputs were moderate in case of mango $(52.30 \mathrm{c})$ and banana $(72.23 \mathrm{c})$, as these were commercially important fruit crops of the region.

Based on the studies conducted, it was found that the farm women had low access to all kinds of resources due to patriarchal nature of the society in which most of the landed properties are inherited by male members and women have poor ownership of land and non-farm assets. Similar findings have been reported by IBRD 2009, who stated that asymmetries in ownership of, access to and control of livelihoods assets (such as land, water, energy, credit, knowledge, and labour) negatively affect woman's food production ability. Differential wage rates of men and women, was another factor which reduced the purchasing ability of women farmers. The time factor is another influential component that hinders women farmers to have access over resources. They are usually overburdened with household chores and care of their children along with addressing the requirements of their male counterparts both at home and at farm. As a result they hardly get time to start their own venture. Poor mobility and accessibility of rural Indian women to various financial, governmental and nongovernmental institutions for financial and nonfinancial services on account of over-burdened household work and existing social structure also widens the gender gap pertaining to their access to resources and deprives them in identifying and assessing their needs for agricultural inputs. FAO 2009 reported that rural women have poor access to resources needed for socio-economic development such as credit, extension, critical inputs, seeds and planting material supply services

\section{Decision making ability}

The decision making ability of farm women with respect to certain issues were also assessed during the study. The criteria of including such aspect in the study were to assess the degree of empowerment of farm women of the region. It was found that the overall participation of farm women in selecting crop/crops of their own choice was moderate $(54.65 \mathrm{c})$. There was overall low participation (43.14 b) in selecting the kind of fruit crops and the varieties to be grown. In situation of surplus yield and glut in the market, the farm women had an overall moderate participation $(49.85 \mathrm{c})$. The results of the study are in confirmation with the findings of Sekar et al., (2014), who reported that women seldom enjoy property ownership rights directly in their names and have little control over decision making in sale of produce, choice of crops, crop production and post-harvest management practices. As a result, men tend to dominate decision-making in both farming and marketing activities.

Considering the crop-wise overall participation of farm women in various activities of fruit cultivation, it can be concluded that the farm women had full participation in cultivation of banana (61.86 d) and moderate participation in mango (51.94 c) and papaya (50.78 c).

The participation was low in fruit crops like litchi (32.85), guava (42.81 b), lemon (37.87 b) and nominal in case of minor fruits $(27.75 \mathrm{a})$. 
From the studies, it was observed that women found Banana as a suitable crop, based on its economy and compact dwarf canopy. While, management of fruit crops like mango and litchi were difficult for farm women on account of huge canopy structure.

Crops like guava, lemon and sapota were grown sparsely in homestead garden, while the minor fruits like jackfruit, custard apple, sapota and jamun were cultivated at very low scale and mostly found neglected.

Farm women are actively involved in many production and post-production activities, which significantly contribute to fruit production. However, due to "Gender issues" and "invisibility" of women's roles, their contribution in this field is not properly acknowledged, which results in wide gender gaps.

The United Nations have identified women and girls empowerment and gender equality as one of their Sustainable Development Goals which highlights the fact that gender equality is imperative for achieving higher, inclusive and sustainable development. Therefore, reducing the gender gaps in fruit cultivation by addressing women's issues and problems will play a significant role in achieving sustainability, prosperity in fruit production along with women's empowerment and livelihood improvement of rural households.

\section{Acknowledgement}

We greatly acknowledge the support and help of Director ICAR-IIHR, Dr. M.R. Dinesh and Head of CHES (ICAR-IIHR) Bhubaneswar, Dr.
H.S. Singh for their valuable support in bringing out this work.

\section{References}

FAO, Gender equity in agriculture and rural development. A quick guide to gender mainstreaming in FAO's new Strategic framework, Rome, Italy, 2009.

Fartyal, S. and Rathore, S. 2013. Vegetable cultivation in Uttarakhand hills: Viewing through a gender lens. Tropical Agricultural Research. 24(3): 238-248.

IBRD, Gender in agriculture. Washington, DC: International Bank for Reconstruction and Development, World Bank, 2009.

Lal, R. and Khurana, A. 2011. Gender issues: the role of women in agriculture sector. Zenith Int. J. Business Economics \& Management Res. 1(1): 29-39.

NSSO, Situation assessment survey of farmers, Ministry of Statistics and Programme Implementation, GoI, 2010.

Oluwatayo, I. B. 2012. Gender considerations in decision making in rural Nigeria: implications on households' food security in Ekiti State; 2008. Retrieved. June 16, 2013. http://erd.eui/media/oluwatayo.pdf on $16 / 06 / 2013$.

Sekar, C., Subramanian, K. S., Subramanian, J., Prakash, V. 2014. Gender dynamics in mango production system in India. Innovare J Soc. Sci., 2(4):74-80.

Sekhar, C., Selvarajan, M., Pounraj, A. and Prahadeeswaran, M. 2013. Production and export of mango in India. American International J. Res. Humanities, Arts Soc. Sci. 13(337): 78-87.

Sethi, R. C. and Sharma, R. B. 2011. Frontier technologies for empowering farm women. Int. J. Farm Sci. 2(1): 142-145.

\section{How to cite this article:}

Sahu Ankita, Kishore Kundan, J.C. Jeeva, S.K. Srivastava and Mohapatra Lalatendu. 2018. Understanding Gender Dynamics in Fruit Cultivation under Indian Condition. Int.J.Curr.Microbiol.App.Sci. 7(10): 1923-1933. doi: https://doi.org/10.20546/ijcmas.2018.710.221 